\title{
Jet Measurements in Heavy lon Collisions with the ATLAS Experiment
}

\author{
Helena Santos, on behalf of the ATLAS Collaboration*t \\ Laboratório de Instrumentação e Física Experimental de Partículas - LIP Portugal \\ E-mail: helena@lip.pt
}

The latest jet measurements in $5.02 \mathrm{TeV} \mathrm{Pb}+\mathrm{Pb}$ collisions with the ATLAS Experiment at the LHC are presented. Jets are direct probes of the quark-gluon plasma produced in heavy-ion collisions as their modifications with respect to jets produced in $p p$ collisions provide insight into their interactions with this QCD medium. The results shown in these proceedings include measurements of nuclear modification factor of jets, jet fragmentation functions, the momentum and angular dependence of charged particle yields within and around jets, and the correlations of jets with direct photons. The presented measurements are also directly compared to stateof-the-art theoretical models and provide important information to understand the strength and mechanism of the jet quenching.

European Physical Society Conference on High Energy Physics - EPS-HEP2019 -

10-17 July, 2019

Ghent, Belgium

\footnotetext{
* Speaker.

${ }^{\dagger}$ The author acknowledges the financial support of Fundação para a Ciência e a Tecnologia (FCT) through FCT contract CERN/FIS-PAR/0008/2017 .
} 


\section{Introduction}

A wide research program is ongoing at the Large Hadron Collider with the aim of studying the properties of QCD matter at extreme temperatures and densities, the quark-gluon plasma (QGP). Jets produced in ultra-relativistic heavy ion collisions constitute a golden probe of such a state of matter. The hard scattered quarks and gluons emerging from these collisions evolve as parton showers that propagate through the hot and dense medium. Constituents of the parton showers emit medium-induced gluon radiation and, as a consequence, the resulting jet loses energy, a phenomenon commonly termed as jet quenching [1]. Jets produced in heavy ion collisions are thus expected to be suppressed at a given $p_{\mathrm{T}}$, relatively to a sample produced in $p p$ collisions. Their internal structure is also expected to be modified. The large acceptance and high granularity of the ATLAS detector [2] is well suited to study these phenomena. Results shown in this conference used data produced in $\mathrm{Pb}+\mathrm{Pb}$ and $p p$ collisions at the center of mass energy of $5.02 \mathrm{TeV}$. The Glauber Monte Carlo model [3] is used to obtain a correspondence between the total transverse energy deposited in the forward calorimeters (FCal, $3.2 \leq|\eta|<4.9$ ) ${ }^{1}$ and the sampling fraction of the total inelastic $\mathrm{Pb}+\mathrm{Pb}$ cross-section, allowing the setting of the centrality percentiles [4]. Jets are reconstructed using calorimeter "towers" as input signals to the anti- $k_{t}$ algorithm, with jet radius parameter size $R=0.4$. The underlying event is estimated and subtracted event-by-event in each calorimeter layer and strip of pseudorapidity after excluding regions underneath jet candidates and corrected for flow modulation [5].

\section{Jet $R_{\mathrm{AA}}$}

The nuclear modification factor $R_{\mathrm{AA}}$, defined as the ratio of normalized yields in $\mathrm{Pb}+\mathrm{Pb}$ and $p p$ interactions, is used to compare the inclusive transverse momentum distributions measured in the two collision systems:

$$
R_{\mathrm{AA}} \equiv \frac{\left(1 / N_{\mathrm{evt}}\right) \mathrm{d}^{2} N_{\mathrm{jet}}^{P b P b} /\left.\mathrm{d} p_{\mathrm{T}} d y\right|_{\mathrm{cent}}}{\left\langle T_{\mathrm{AA}}\right\rangle \mathrm{d}^{2} \sigma_{\text {jet }}^{p p} / \mathrm{d} p_{\mathrm{T}} d y}
$$

where $\left\langle T_{\mathrm{AA}}\right\rangle$ stands for the geometric enhancement of per-collision nucleon-nucleon luminosity and $N_{\text {evt }}$ is the total number of $\mathrm{Pb}+\mathrm{Pb}$ collisions within a chosen centrality interval (cent). The nuclear modification factor $R_{\mathrm{AA}}$, resulting from distributions unfolded for detector resolution, binto-bin migration and reconstruction inefficiency, is shown in Figure 1. Jets are suppressed by a factor of two in central collisions ( $0-10 \%)$, while showing clear dependence on jet transverse momentum [6]. These measurements confirm the expectations on the reduction of the jet yields at a given transverse momentum due to the interaction of partons in the QGP [1]. The Linear Boltzmann Transport model, that combines kinetic description of parton propagation with hydrodynamic description of the underlying medium evolution, reproduces very reasonably both the trend and magnitude of the $R_{\mathrm{AA}}[7]$.

\footnotetext{
${ }^{1}$ ATLAS uses a right-handed coordinate system with its origin at the nominal interaction point (IP) in the centre of the detector and the z-axis along the beam pipe. The x-axis points from the IP to the centre of the LHC ring, and the y-axis points upwards. Cylindrical coordinates $(r, \phi)$ are used in the transverse plane, $\phi$ being the azimuthal angle around the z-axis. The pseudorapidity is defined in terms of the polar angle $\theta$ as $\eta=-\ln \tan (\theta / 2)$.
} 


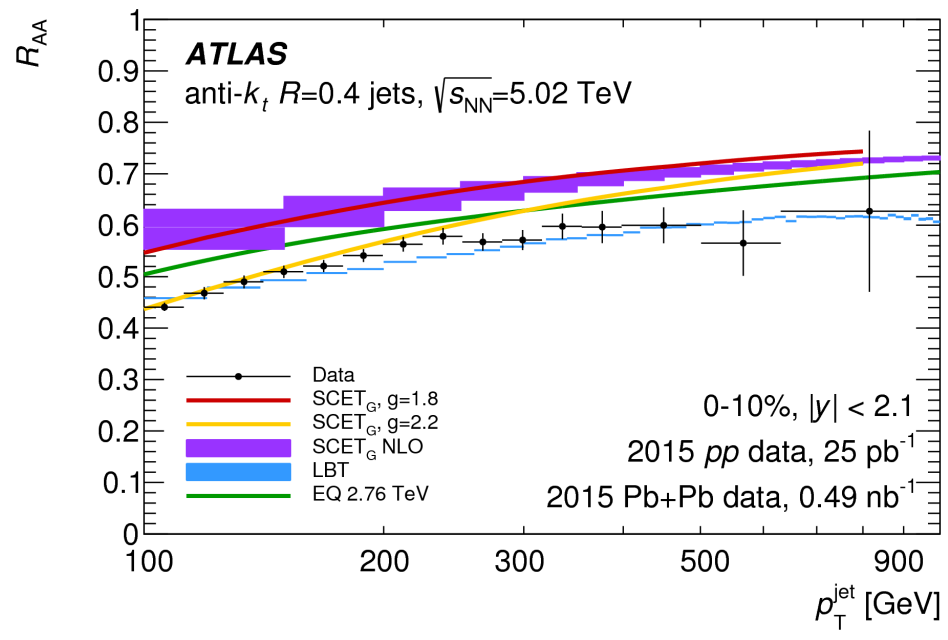

Figure 1: The $R_{\mathrm{AA}}$ as a function of jet $p_{\mathrm{T}}$ for jets produced in central collisions (0-10\%) and with $|y|<2.1$ compared with theory predictions $[7,8,9]$. The uncertainties of the data points are the combined statistical and systematic uncertainties [6]. The vertical width of the distribution shown for the LBT and SCET ${ }_{G}$ NLO $^{-}$ models represents the uncertainty of the theory prediction.

\section{Jet Fragmentation Functions}

Jet fragmentation functions are measured with the expectation of getting a deeper understanding of the jet energy loss nature and constraining jet quenching models. The jet structure is probed in the $126-398 \mathrm{GeV} p_{\mathrm{T}}$ range, with an absolute value of jet rapidity less than 2.1 , and using tracks with $p_{\mathrm{T}}>1 \mathrm{GeV}$. The fragmentation functions, defined as $D\left[z\left(p_{\mathrm{T}}\right)\right]=\left(1 / N_{\mathrm{jet}}\right) d N_{\mathrm{ch}} / d z\left(p_{\mathrm{T}}\right)$, are studied as a function of the longitudinal momentum fraction, $z=p_{\mathrm{T}}{ }^{\mathrm{ch}} / p_{\mathrm{T}}{ }^{\mathrm{jet}} \cos \Delta R$, and track $p_{\mathrm{T}}$, where $p_{\mathrm{T}}{ }^{\text {ch }}$ stands for the transverse momentum of a charged particle and $\Delta R$ is the distance between the charged particle and the jet axis. The $D(z)$ and $D\left(p_{\mathrm{T}}\right)$ distributions are background subtracted, corrected for reconstruction inefficiency and unfolded with a two-dimensional Bayesian method in $z$, or $p_{\mathrm{T}}{ }^{\text {ch }}$, and $p_{\mathrm{T}}{ }^{\text {jet }}$. The left panel of Figure 2 shows the modification of the fragmentation functions in central collisions (0-10\%), assessed with $R_{D(z)}=\left.D(z)\right|_{\mathrm{Pb}+\mathrm{Pb}} /\left.D(z)\right|_{\mathrm{pp}}$ in different ranges of jet transverse momentum, $p_{\mathrm{T}}{ }^{\text {jet }}$. An enhancement at low and high $z$ is observed, whereas the range $0.01<z<0.2$ shows a clear depletion. The enhancement of fragment yields at low $z$ and depletion at intermediate $z$ are consistent with a model which uses calculations based on soft collinear effective theory (SCET) $[8,10]$. This model does not reproduce the enhancement in the high-z region observed in the data, though. The right plot shows similar distributions but in the variable $p_{\mathrm{T}}$ of the charged particle within the jet. The observed enhancement of high- $p_{\mathrm{T}}$ particles within jets with $p_{\mathrm{T}}$ in the ranges $126-158 \mathrm{GeV}$ and $200-251 \mathrm{GeV}$ is in agreement with a hybrid model of jet quenching, which uses perturbative techniques for the high- $Q^{2}$ processes in jet evolution and strong coupling for the low momentum scales associated with the QGP [11]. However, this model does not reproduce the data at the low- $p_{\mathrm{T}}$ range. Details on this analysis can be found in [12].

The study of the fragmentation functions as a function of the angular distance, $r$, between the charged particle and the jet axis allows to understand how the particles are re-distributed in order 

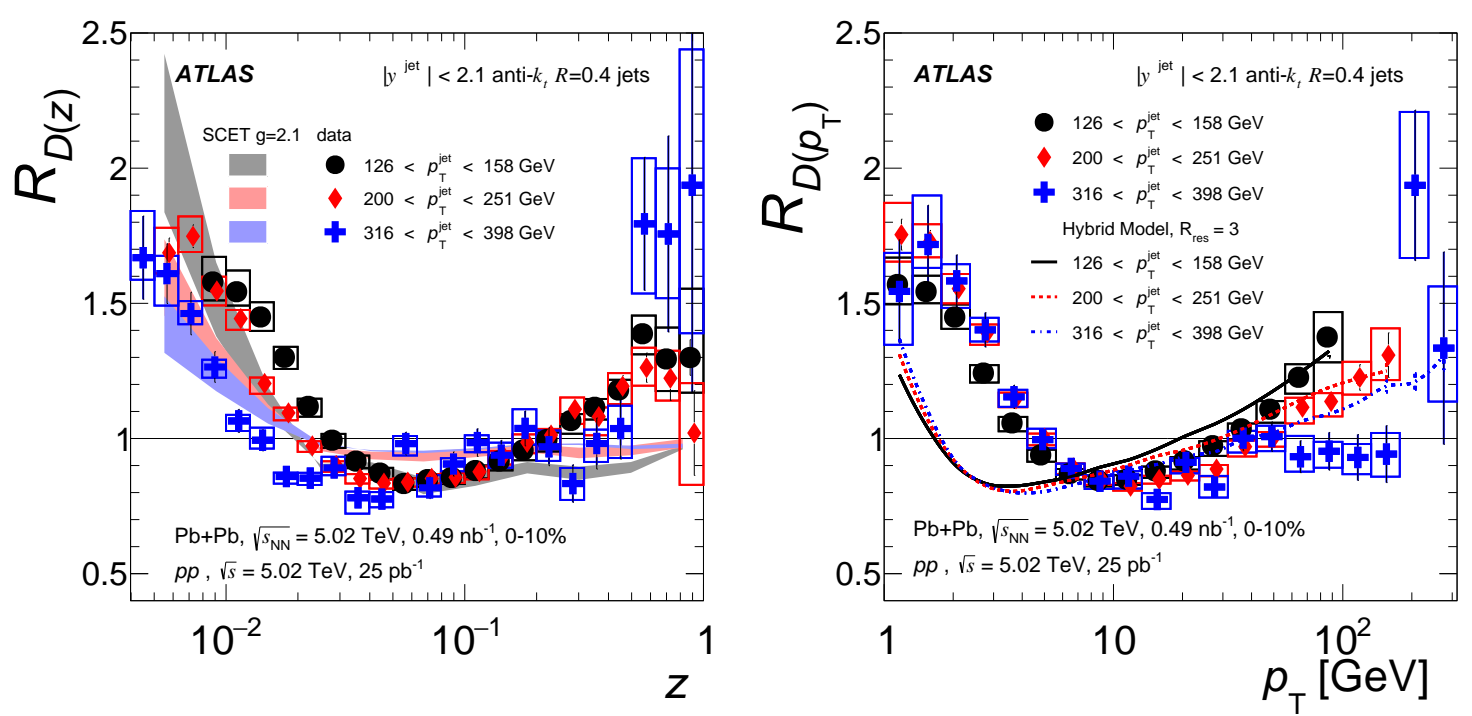

Figure 2: Left panel: $R_{D(z)}$ for three $p_{\mathrm{T}}^{\text {jet }}$ ranges: $126-158 \mathrm{GeV}$ (circles), 200-251 GeV (diamonds) and $316-398 \mathrm{GeV}$ (crosses) compared with calculations from the SCET model [8,10]. Right panel: $D\left(p_{\mathrm{T}}\right)$ ratios for the same three $p_{\mathrm{T}}{ }^{\text {jet }}$ ranges compared with calculations from the hybrid model with $\mathrm{R}_{\text {res }}=3$ [11]. See ref. [12].

to accommodate the observed modification at a given $p_{\mathrm{T}}$. In this analysis, charged particles with transverse momentum in the $1-63 \mathrm{GeV}$ range are matched to jets with transverse momentum in the $126-316 \mathrm{GeV}$ range and an absolute value of jet rapidity of less than 1.7 [13]. In central $\mathrm{Pb}+\mathrm{Pb}$ collisions, yields of charged particles with $p_{\mathrm{T}}$ lower than $4 \mathrm{GeV}$ are observed to be increasingly enhanced as a function of angular distance from the jet axis, reaching a maximum at $r=0.5$, as can be seen in the left panel of Figure 3. On the contrary, particles with transverse momentum above 4 $\mathrm{GeV}$ have an enhanced yield in the jet core for angular distances up to $r=0.05$ from the jet axis, with a suppression at larger distances. This behaviour is compatible with the inclusive jet fragmentation functions shown above, indicating that the enhanced number of low- $p_{\mathrm{T}}$ particles shown in the right plot of Figure 2 dominates at larger radial distances. The modifications are shown to be centrality dependent, decreasing for more peripheral collisions (right panel).

\section{4. $\gamma$-Jet correlations}

A suitable observable to probe jet energy loss is the jet energy relative to photon energy, as photons do not experience the strong interaction and will emerge untouched from the fireball. The transverse momentum and pseudorapidity cuts applied to photons in the current analysis are $63.1<p_{\mathrm{T}}^{\gamma}<200 \mathrm{GeV}$ and $\left|\eta_{\gamma}\right|<2.37$, while for jets are ${p_{\mathrm{T}}}^{\text {jet }}>31.6 \mathrm{GeV}$ and $\left|\eta_{\text {Jet }}\right|<2.8$. The transverse momentum balance given by the jet-to-photon $p_{\mathrm{T}}$ ratio, $x_{\mathrm{J} \gamma}$, is measured for pairs with azimuthal opening angle $\Delta \phi>7 \pi / 8$. Figure 4 shows the distributions of the per-photon jet yield as a function of $x_{\mathrm{J} \gamma},\left(1 / N_{\gamma}\right)\left(d N / x_{\mathrm{J} \gamma}\right)$, fully corrected for detector effects using a twodimensional unfolding procedure and reported at the particle level. The top set of plots concerns the $p_{\mathrm{T}}$ of the photon in the $63.1-79.6 \mathrm{GeV}$ range and the bottom set concerns the $100-158 \mathrm{GeV}$ $p_{\mathrm{T}}$ range. Whereas there is a $p p$-like peaked $x_{\mathrm{J} \gamma}$, independently of jet $p_{\mathrm{T}}$ in peripheral $\mathrm{Pb}+\mathrm{Pb}, \mathrm{a}$ 

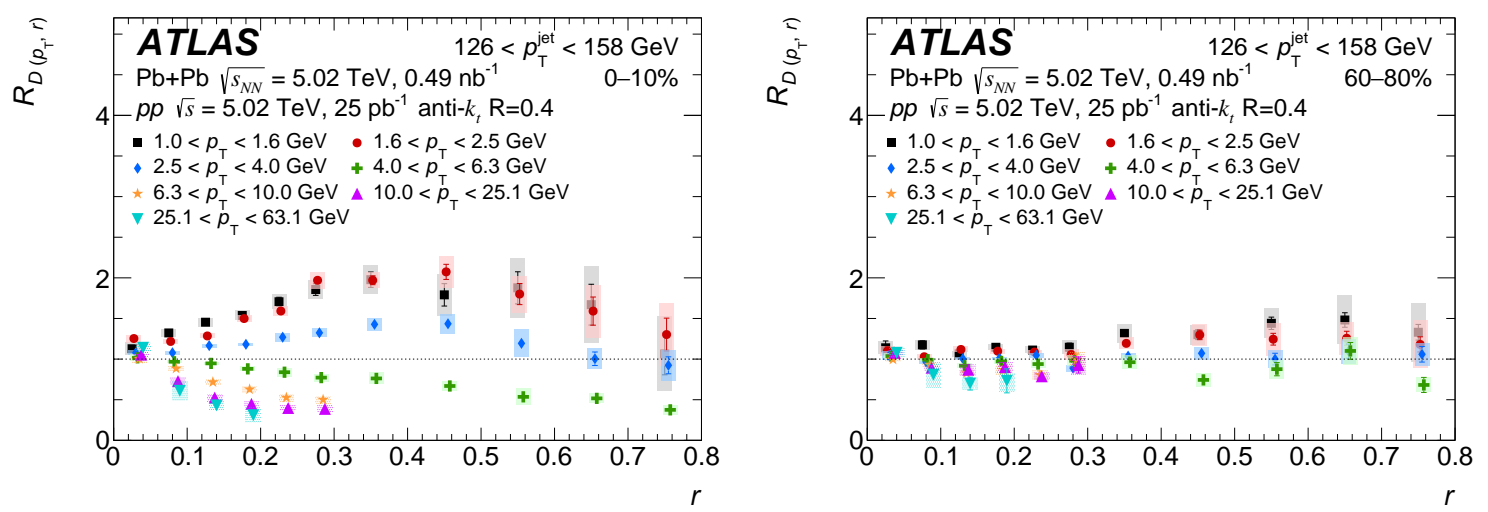

Figure 3: Ratios of $D\left(p_{\mathrm{T}}, r\right)$ distributions in $\mathrm{Pb}+\mathrm{Pb}$ and $p p$ collisions as a function of angular distance $r$ for $126<p_{\mathrm{T}}{ }^{\text {jet }}<158 \mathrm{GeV}$ (left) and for seven $p_{\mathrm{T}}{ }^{\text {ch }}$ selections. Different centrality selections are shown: $0-10 \%$ (left panel) and 60-80\% (right panel). The vertical bars on the data points indicate statistical uncertainties, while the shaded boxes indicate systematic uncertainties. The widths of the boxes are not indicative of the bin size, and the points are shifted horizontally for better visibility. [13].

striking structure evolving with collision centrality and sensitive to jet $p_{\mathrm{T}}$ is observed. Noticeable is the increasing double peak, interpreted as resulting from parton energy loss in the hot nuclear medium [14].

\section{Conclusions}

Inclusive jets yields are found to be suppressed by a factor of two in central collisions, while showing significant dependence on jet transverse momentum. The Linear Boltzmann Transport model reproduces both the trend and magnitude of the suppression. The jet structure is modified: in central collisions there is an enhancement of particles at low and high $z$ or $p_{\mathrm{T}}{ }^{\mathrm{ch}}$, while a suppression at intermediate $z\left(p_{\mathrm{T}}{ }^{\mathrm{ch}}\right)$ is observed. Models confirm partially the shape of these modifications. The $R_{D(p T, r)}$ distributions for charged particles with $p_{\mathrm{T}}<4 \mathrm{GeV}$ are above unity, whereas charged particles with $p_{\mathrm{T}}>4 \mathrm{GeV}$ are suppressed. Such a behaviour confirms the modifications of the inclusive jet fragmentation functions. The photon-jet $p_{\mathrm{T}}$ balance, $x_{\mathrm{J} \gamma}$, distributions are observed to be modified when compared with those in $p p$ collisions. These modifications have a smooth onset as a function of collisions centrality and $p_{\mathrm{T}}^{\gamma}$.

\section{References}

[1] J.-P. Blaizot and Y. Mehtar-Tani, J. Mod. Phys. E24, 1530012 (2015) (and references therein). arXiv: 1503.05958 [hep-ph].

[2] ATLAS Collaboration, JINST 3, S08003 (2008).

[3] M.L. Miller, K. Reygers, S.J. Sanders, and P. Steinberg, Ann. Rev. Nucl. Part. Sci. 57, 205 (2007).

[4] ATLAS Collaboration, Phys. Lett. B 707, 330 (2012). arXiv:1108.6018 [hep-ex]. 


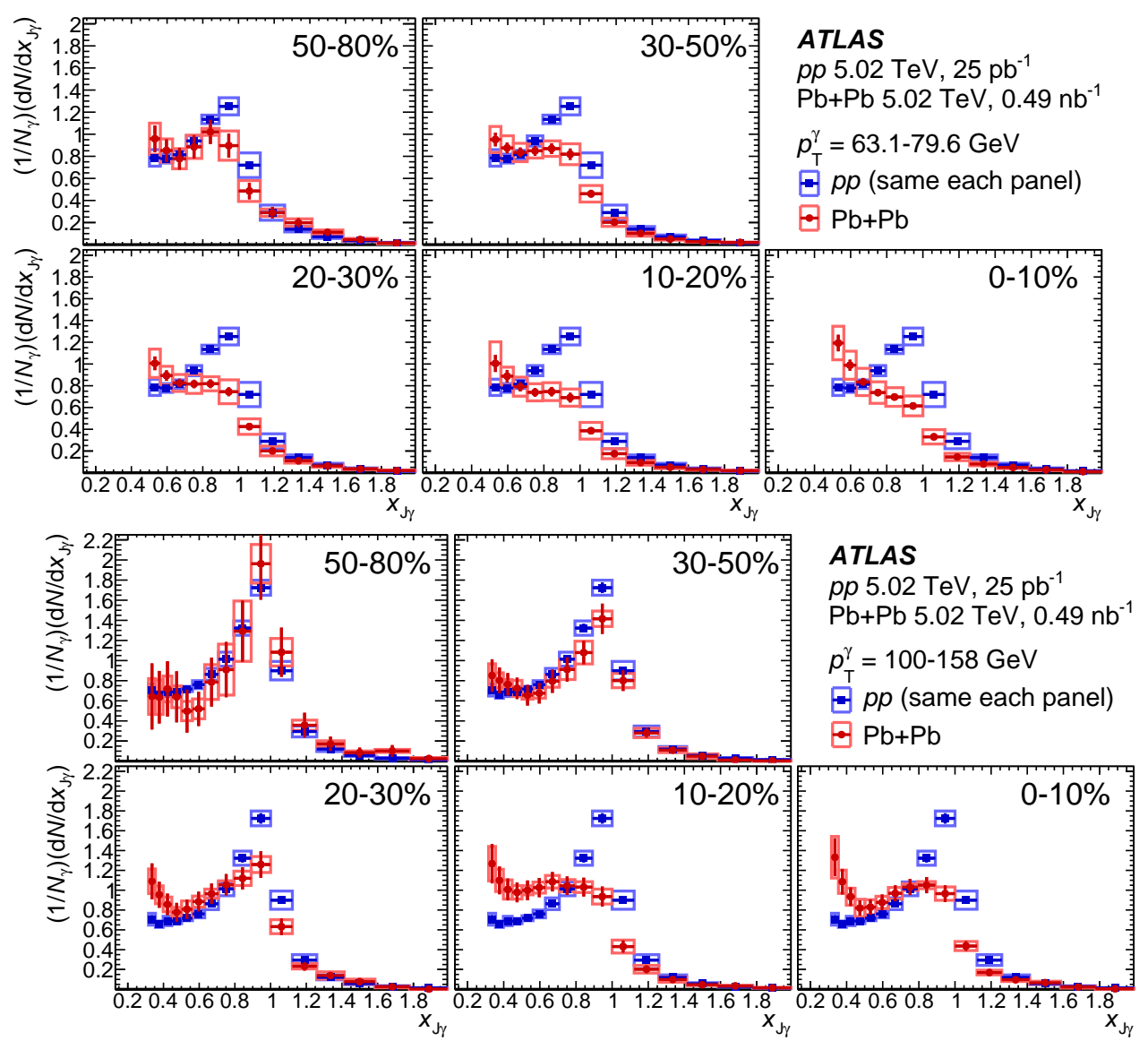

Figure 4: Fully unfolded and corrected photon-jet $p_{\mathrm{T}}$-balance distributions, $\left(1 / N_{\gamma}\right)\left(d N / x_{\mathrm{J}}\right)$, in $\mathrm{Pb}+\mathrm{Pb}$ events (red circles) with each panel showing a different centrality selection compared to that in $p p$ events (blue squares). Total systematic uncertainties are shown as boxes, while statistical uncertainties are shown as vertical bars. Top: results for $p_{\mathrm{T}}^{\gamma}=63.1-79.6 \mathrm{GeV}$. Bottom: results for $p_{\mathrm{T}}^{\gamma}=100-158 \mathrm{GeV}$ [14].

[5] ATLAS Collaboration, Phys. Rev. Lett. 114, 072302 (2015). arXiv:1411.2357v2 [hep-ex].

[6] ATLAS Collaboration, Phys. Lett. B 790, 108 (2019). arXiv:1805.05635 [nucl-ex].

[7] Y. He, T. Luo, X.-N. Wang and Y. Zhu, Phys. Rev. C 91, 054908 (2015). arXiv:1503.03313 [nucl-th].

[8] Y.-T. Chien, A. Emerman, Z.-B. Kang, G. Ovanesyan, and I. Vitev, Phys. Rev. D 93, 074030 (2016). arXiv:1509.02936 [hep-ph].

[9] M. Spousta, B. Cole, Eur.Phys.J. C76 (2016) 50. arXiv:1504.05169 [hep-ph].

[10] Z.-B. Kang, F. Ringer, and I. Vitev, Phys. Lett. B 769, 242 (2017).

[11] Z. Hulcher, D. Pablos, and K. Rajagopal, J. High Energy Phys. 03, 010 (2018).

[12] ATLAS Collaboration, Phys. Rev. C 98, 024908 (2018). arXiv:1805.05424 [hep-ex].

[13] ATLAS Collaboration, arXiv:1908.05264 [hep-ex].

[14] ATLAS Collaboration, Phys. Rev. Lett. 123, 042001 (2019). arXiv:1902.10007 [hep-ex]. 